Supplement of Geosci. Model Dev., 12, 1525-1539, 2019

https://doi.org/10.5194/gmd-12-1525-2019-supplement

(C) Author(s) 2019. This work is distributed under

the Creative Commons Attribution 4.0 License.

(c) (1)

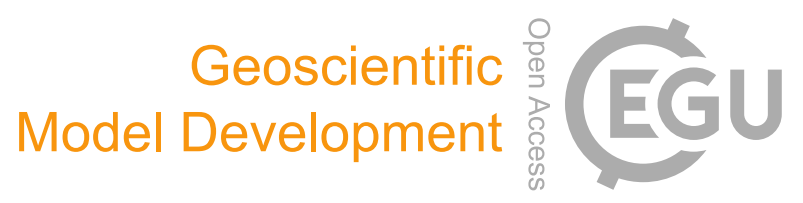

Supplement of

Scalable diagnostics for global atmospheric chemistry using Ristretto library (version 1.0)

Meghana Velegar et al.

Correspondence to: J. Nathan Kutz (kutz@uw.edu)

The copyright of individual parts of the supplement might differ from the CC BY 4.0 License. 


\section{Additional Diagnostics}

We present results for 5 more chemicals of interest in this supplement:

$-\log (\mathbf{N O})$

$-\log \left(\mathbf{N O}_{2}\right)$

- OH

$-\log (\mathbf{I S O P})$

- CO

These chemical species are known to dominate the dynamics on the global scale. Shown are the various matrix decompositions 15 for each species. They can be compared with the species represented in the main text body.

\section{Computational Results for Various Dominant Chemical Species}

Figures 1 to 6 represent the singular value spectrum and modes for the three matrix decompositions presented in the main body of the text for Nitric Oxide.

Figures 7 to 12 represent the singular value spectrum and modes for the three matrix decompositions presented in the main 20 body of the text for Nitrous Dioxide.

Figures 13 to 18 represent the singular value spectrum and modes for the three matrix decompositions presented in the main body of the text for Hydroxyl ion $\mathbf{O H}$.

Figures 19 to 24 represent the singular value spectrum and modes for the three matrix decompositions presented in the main body of the text for Isoprene data $\log ($ ISOP).

Figures 25 to 30 represent the singular value spectrum and modes for the three matrix decompositions presented in the main body of the text for Carbon monoxide $\mathbf{C O}$ 

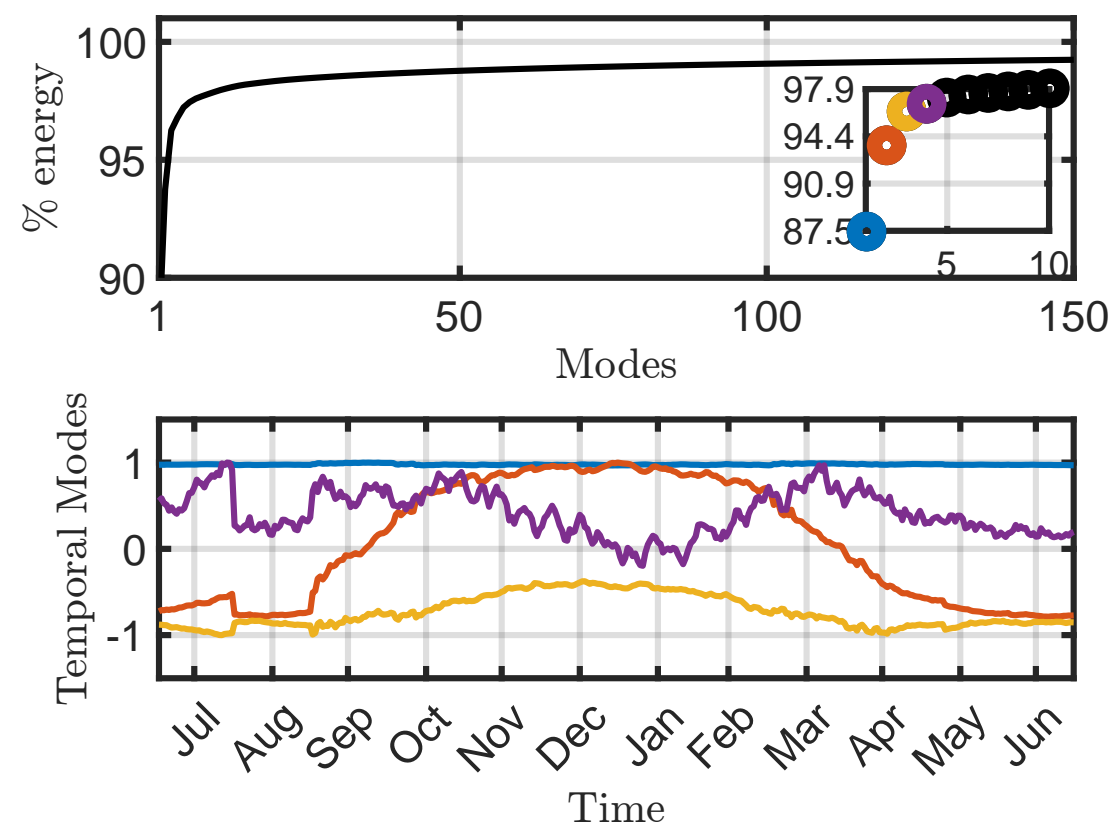

Figure 1. Singular values on a log scale (top) and the corresponding 4 dominant temporal modes (bottom) for $\log (\mathrm{NO})$ absolute concentration preprocessed data.

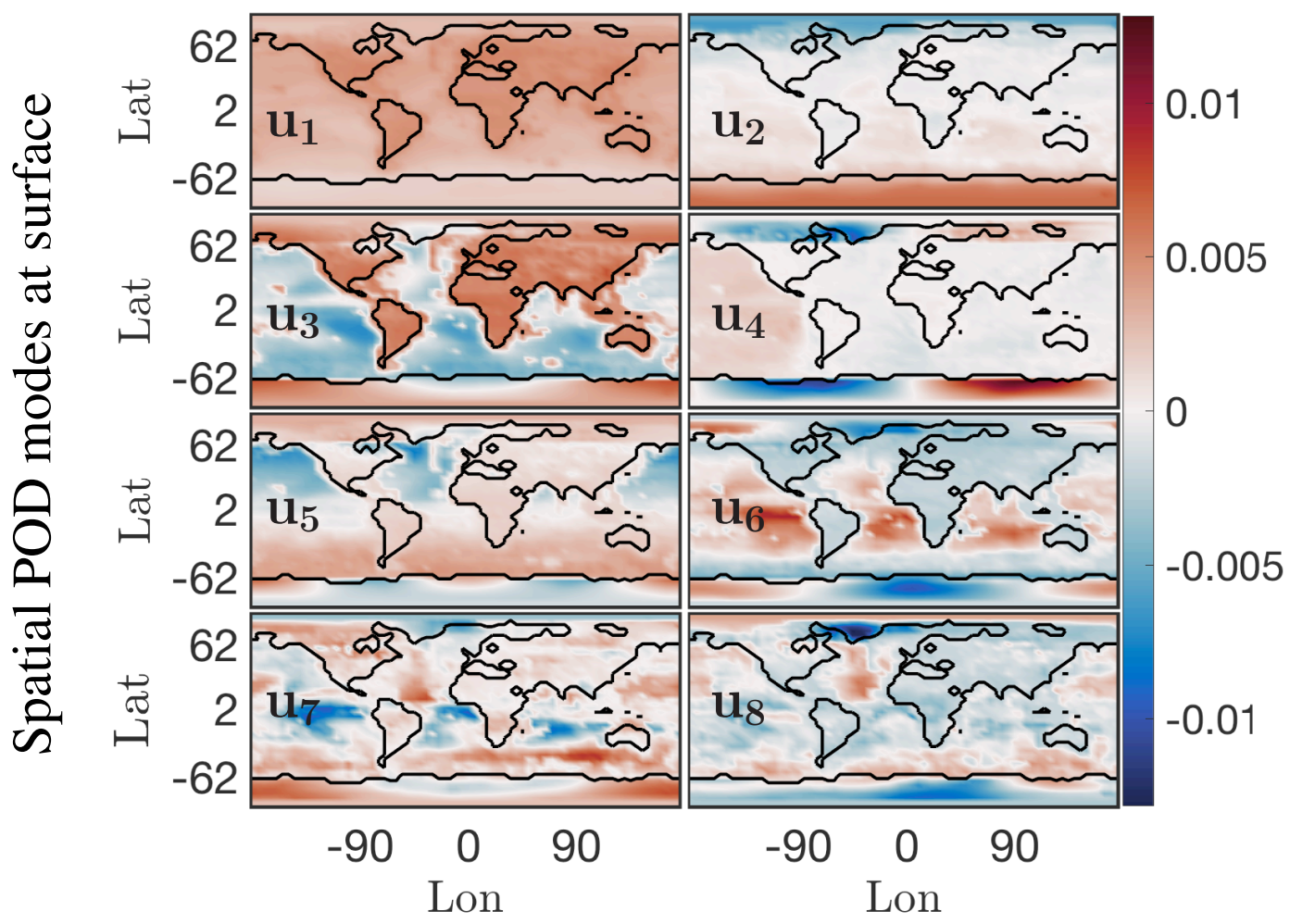

Figure 2. First 8 dominant spatial modes at surface for $\log (\mathrm{NO})$ absolute concentration preprocessed data 

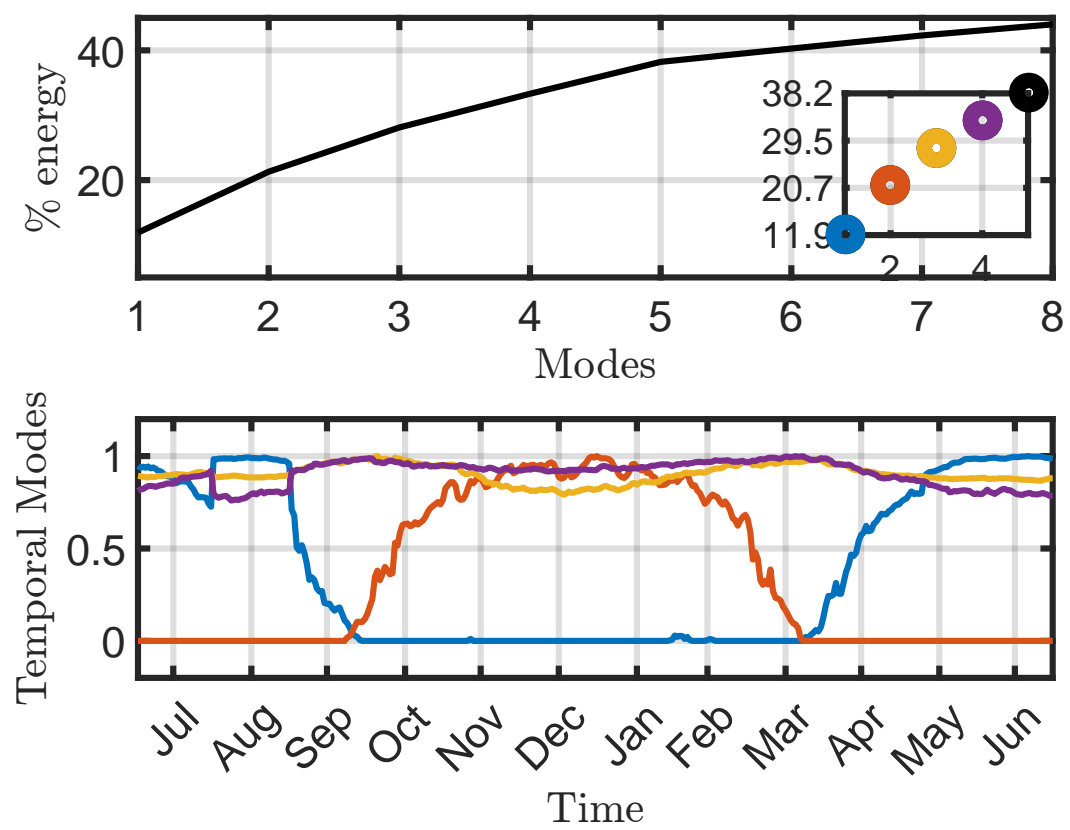

Figure 3. Cumulative energy spectrum from the Nonnegative Matrix Factorization (top) and the corresponding first 4 columns of the ordered $\mathbf{H}$ temporal factor for $\log (\mathrm{NO})$ absolute concentration preprocessed data (bottom).

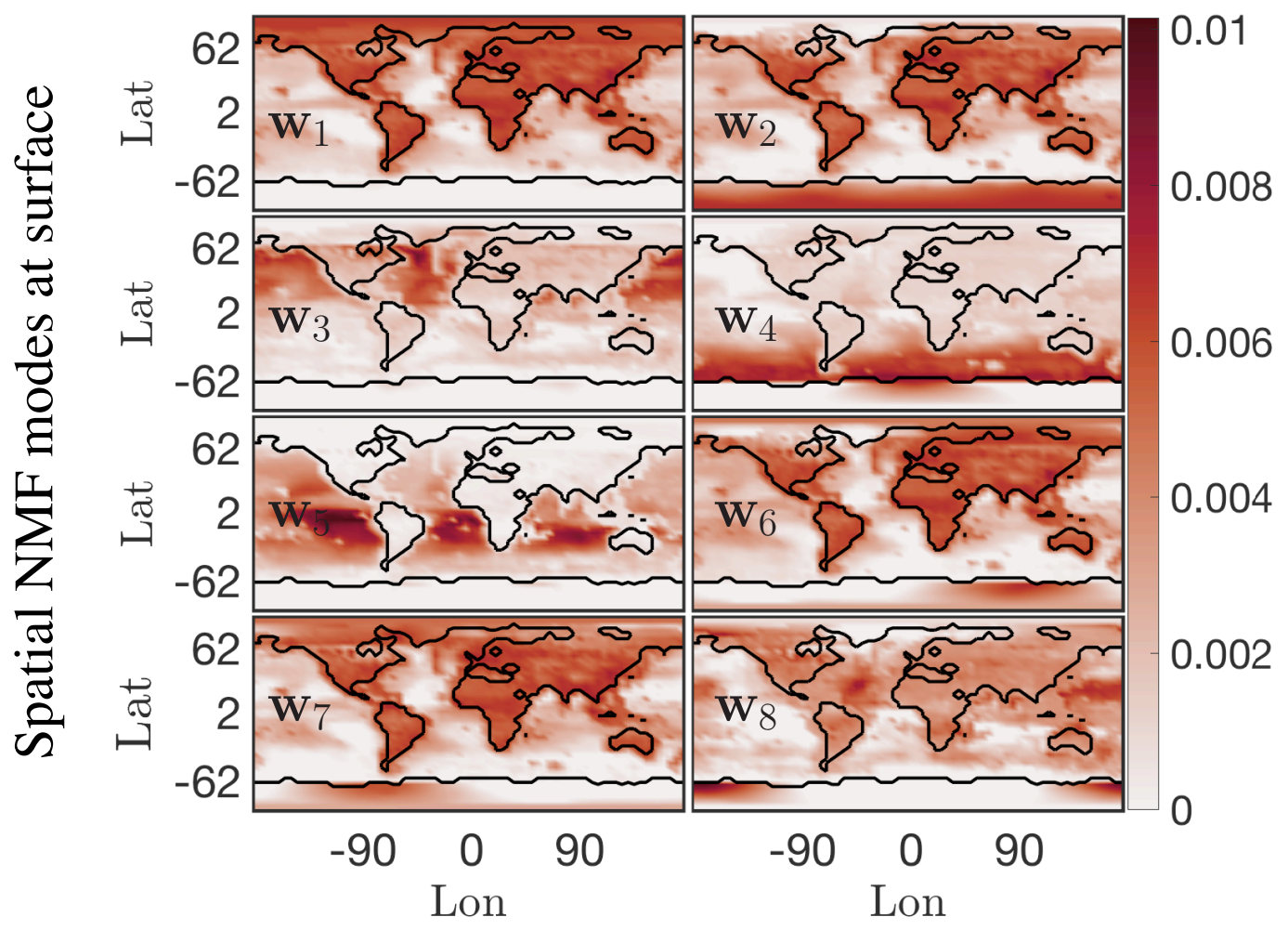

Figure 4. First 8 columns of ordered $\mathbf{W}$ spatial factor from NMF at surface for $\log (\mathrm{NO})$ absolute concentration preprocessed data 

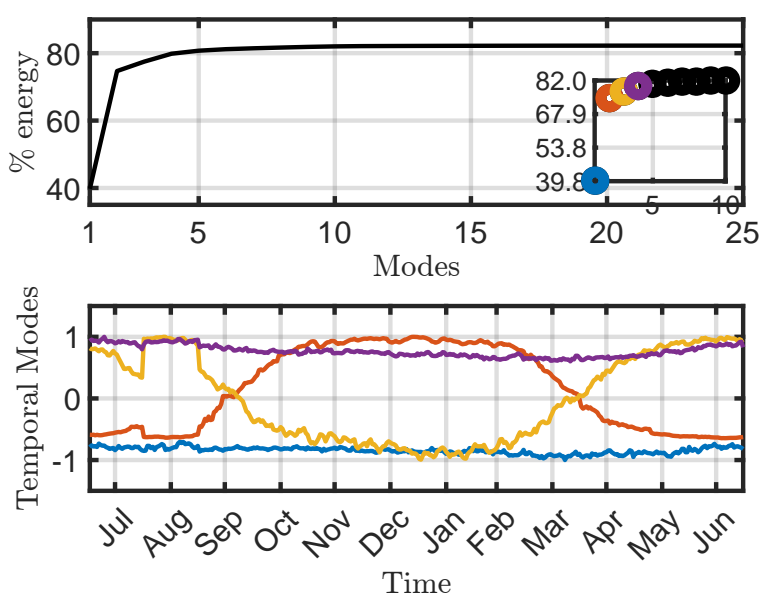

Figure 5. Cumulative energy spectrum from the Sparse Principal Component Anaylsis (top) and the corresponding 4 dominant temporal modes (bottom) for $\log (\mathbf{N O})$ absolute concentration preprocessed data.

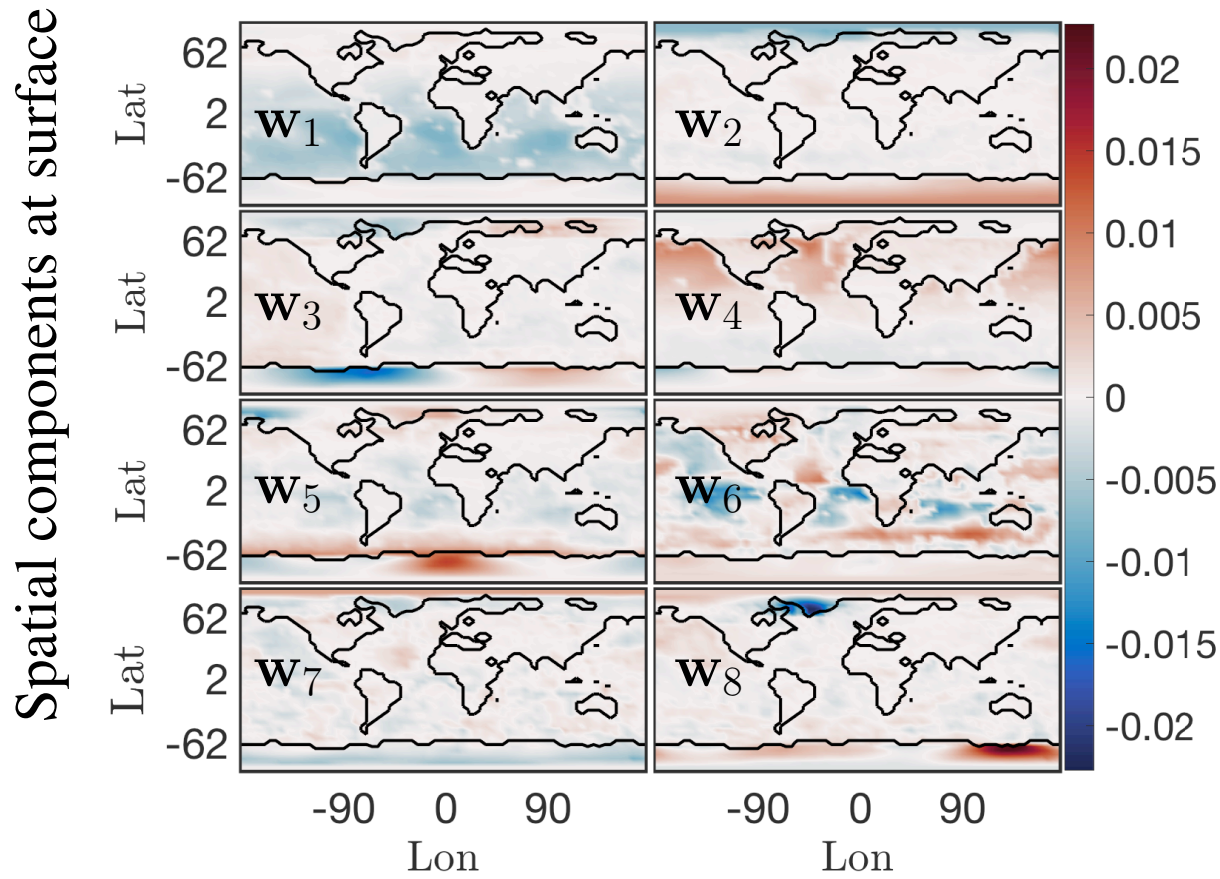

Figure 6. First 8 principal components from SPCA at surface for $\log (\mathrm{NO})$ absolute concentration preprocessed data 

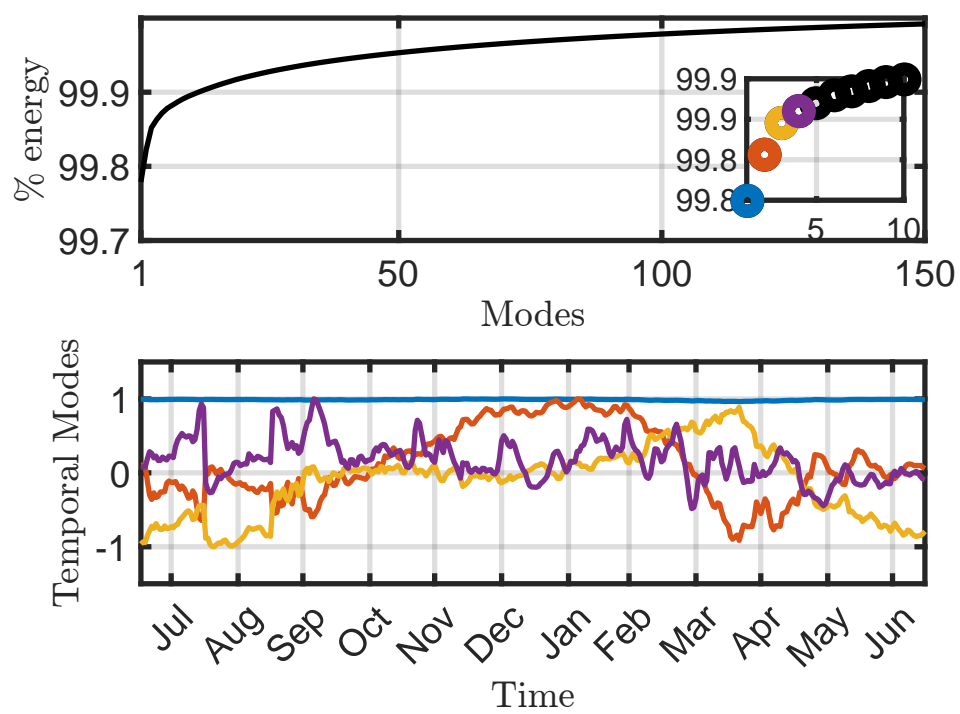

Figure 7. Singular values on a log scale (top) and the corresponding 4 dominant temporal modes (bottom) for $\log \left(\mathbf{N O}_{2}\right)$ absolute concentration preprocessed data.

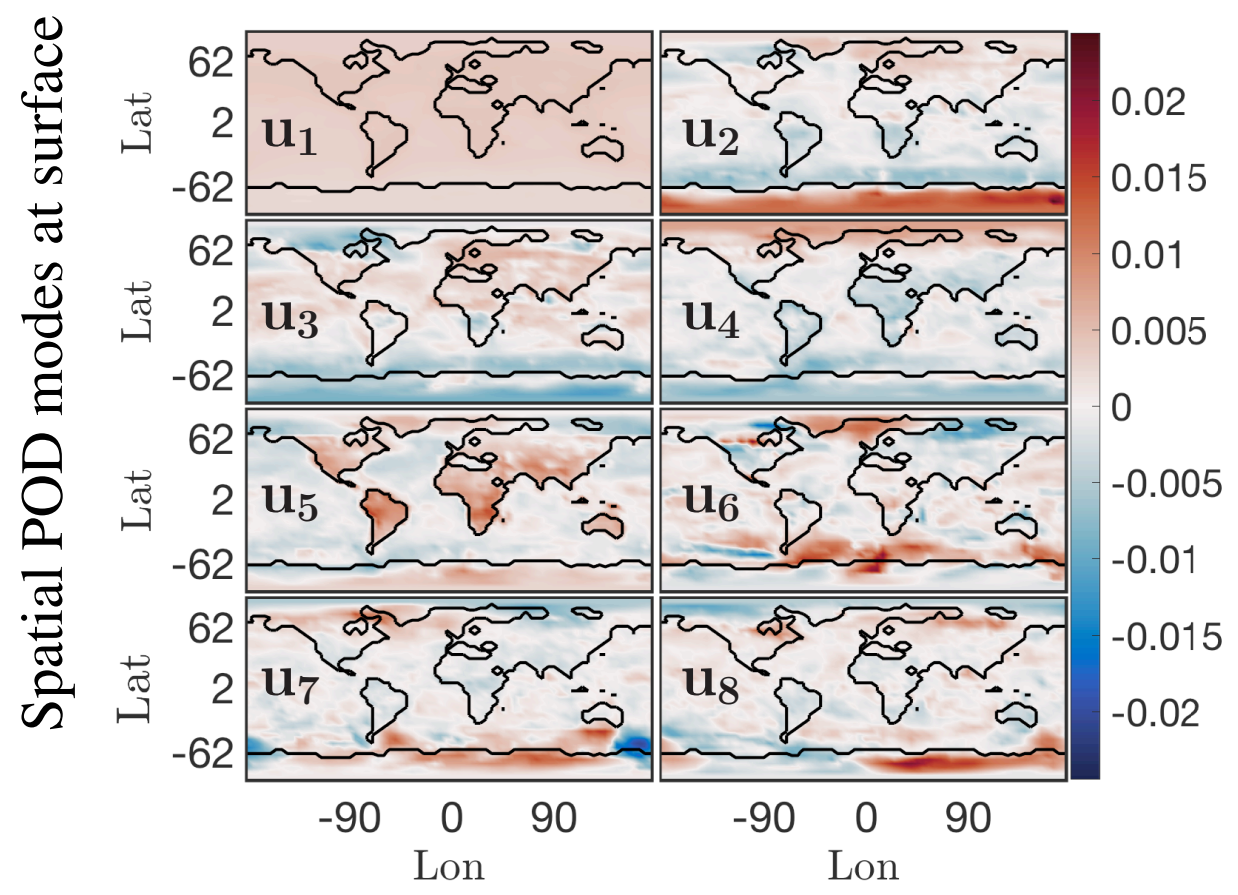

Figure 8. First 8 dominant spatial modes at surface for $\log \left(\mathrm{NO}_{2}\right)$ absolute concentration preprocessed data 

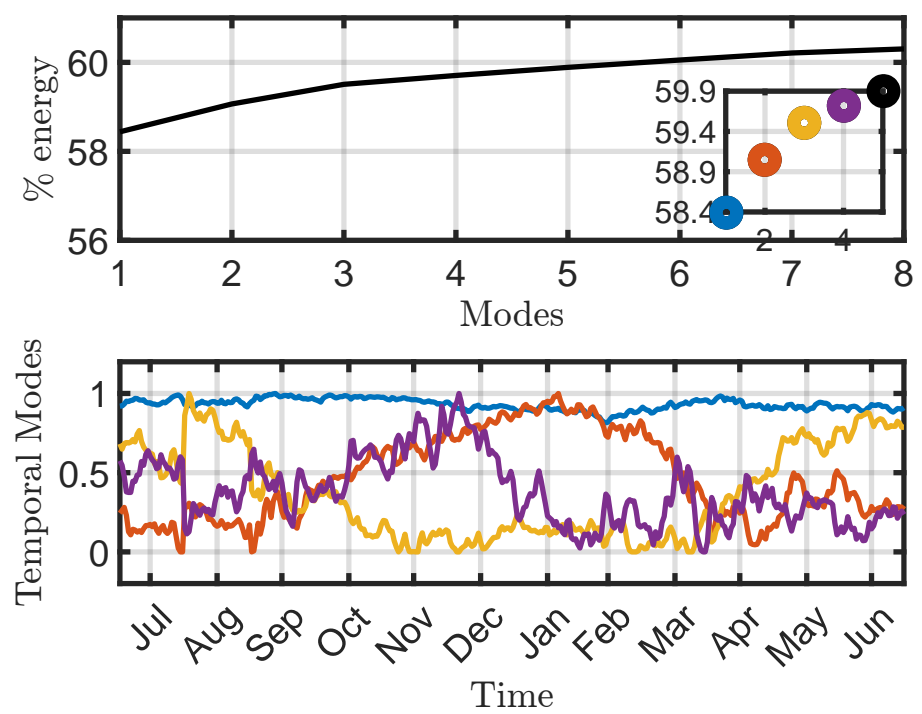

Figure 9. Cumulative energy spectrum from the Nonnegative Matrix Factorization (top) and the corresponding first 4 columns of the ordered $\mathbf{H}$ temporal factor for $\log \left(\mathbf{N O}_{2}\right)$ absolute concentration preprocessed data (bottom).

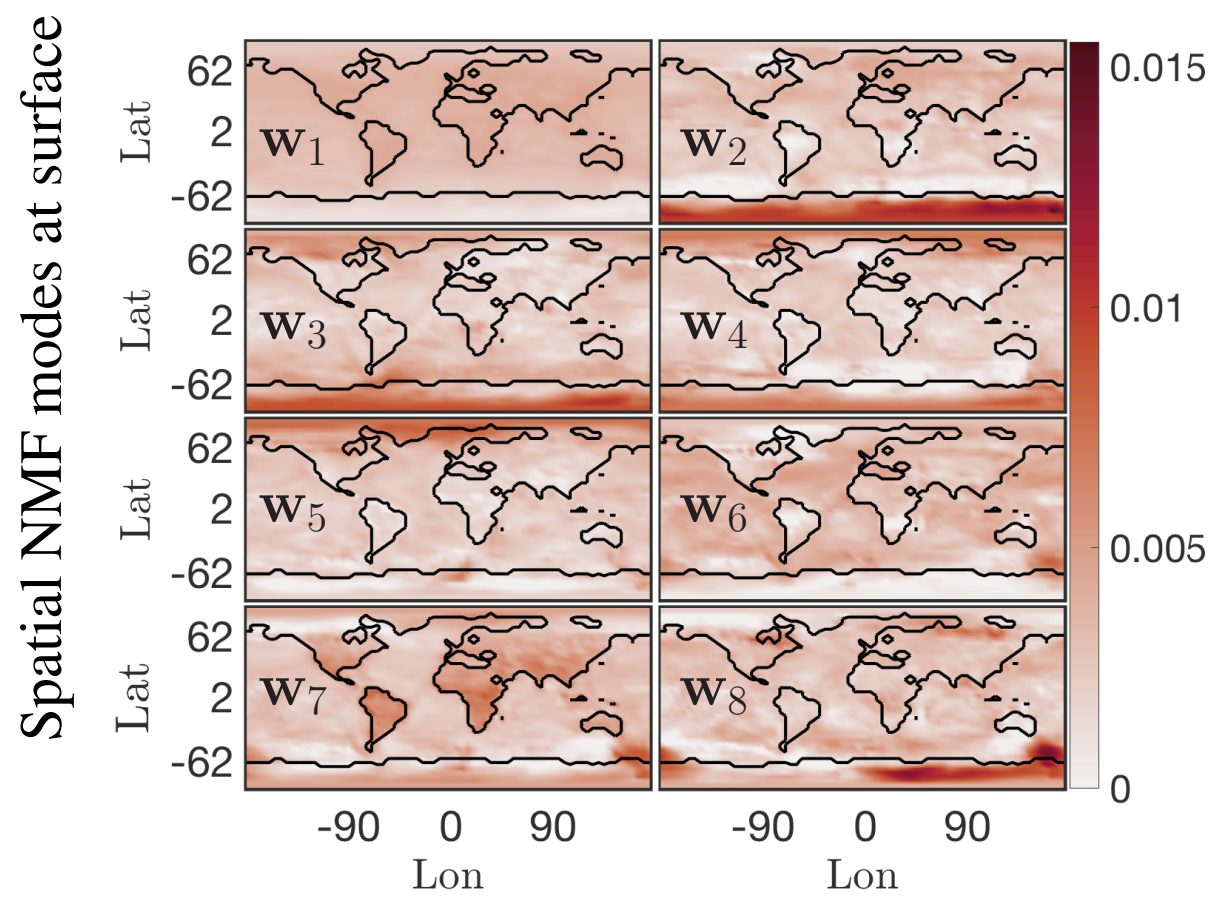

Figure 10. First 8 columns of ordered $\mathbf{W}$ spatial factor from $N M F$ at surface for $\log \left(\mathrm{NO}_{2}\right)$ absolute concentration preprocessed data 

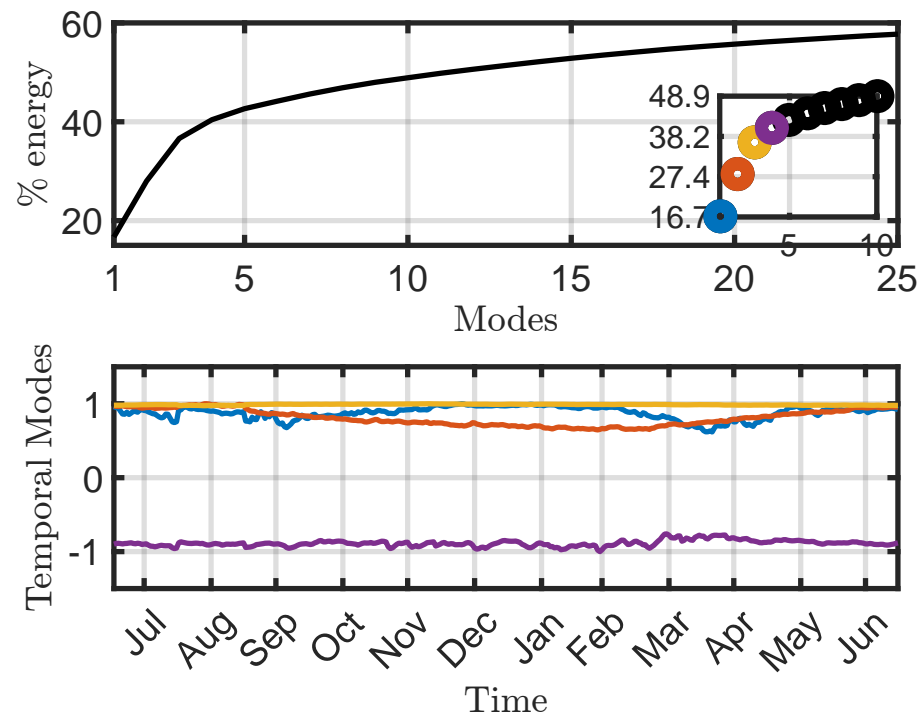

Figure 11. Cumulative energy spectrum from the Sparse Principal Component Anaylsis (top) and the corresponding 4 dominant temporal modes (bottom) for $\log \left(\mathrm{NO}_{2}\right)$ absolute concentration preprocessed data.

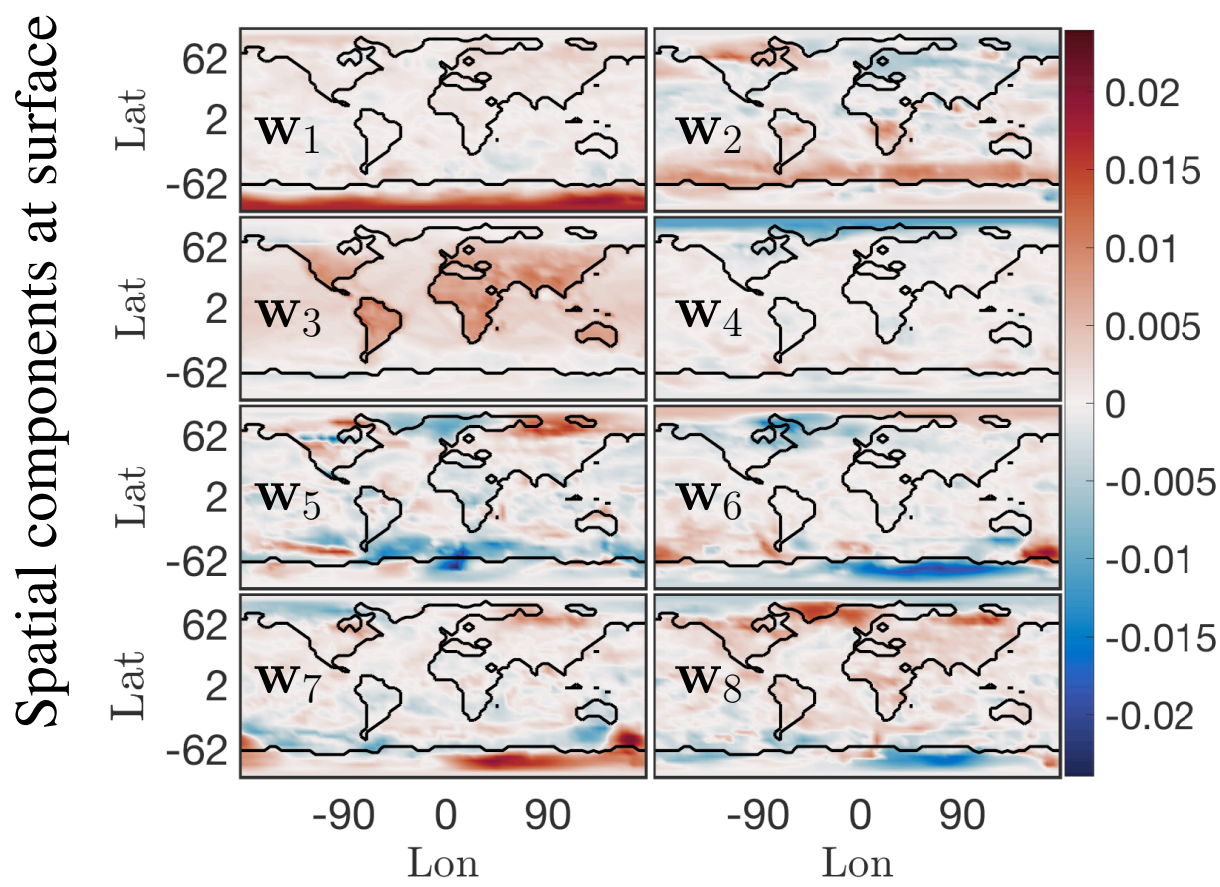

Figure 12. First 8 principal components from SPCA at surface for $\log \left(\mathrm{NO}_{2}\right)$ absolute concentration preprocessed data 

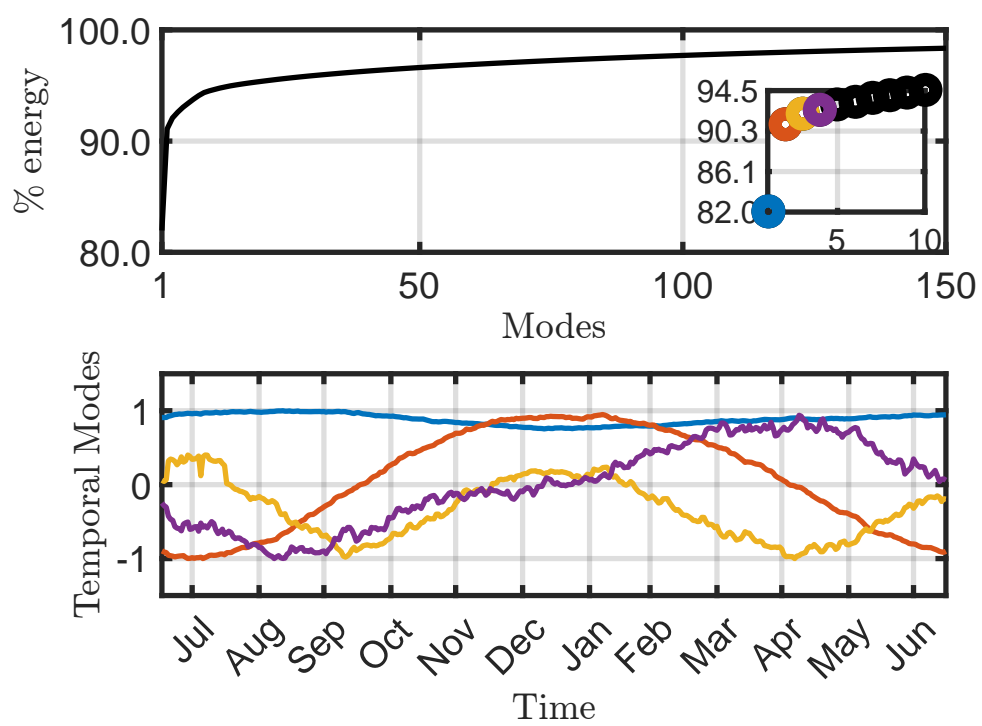

Figure 13. Singular values on a log scale (top) and the corresponding 4 dominant temporal modes (bottom) for $\mathbf{O H}$ absolute concentration preprocessed data.

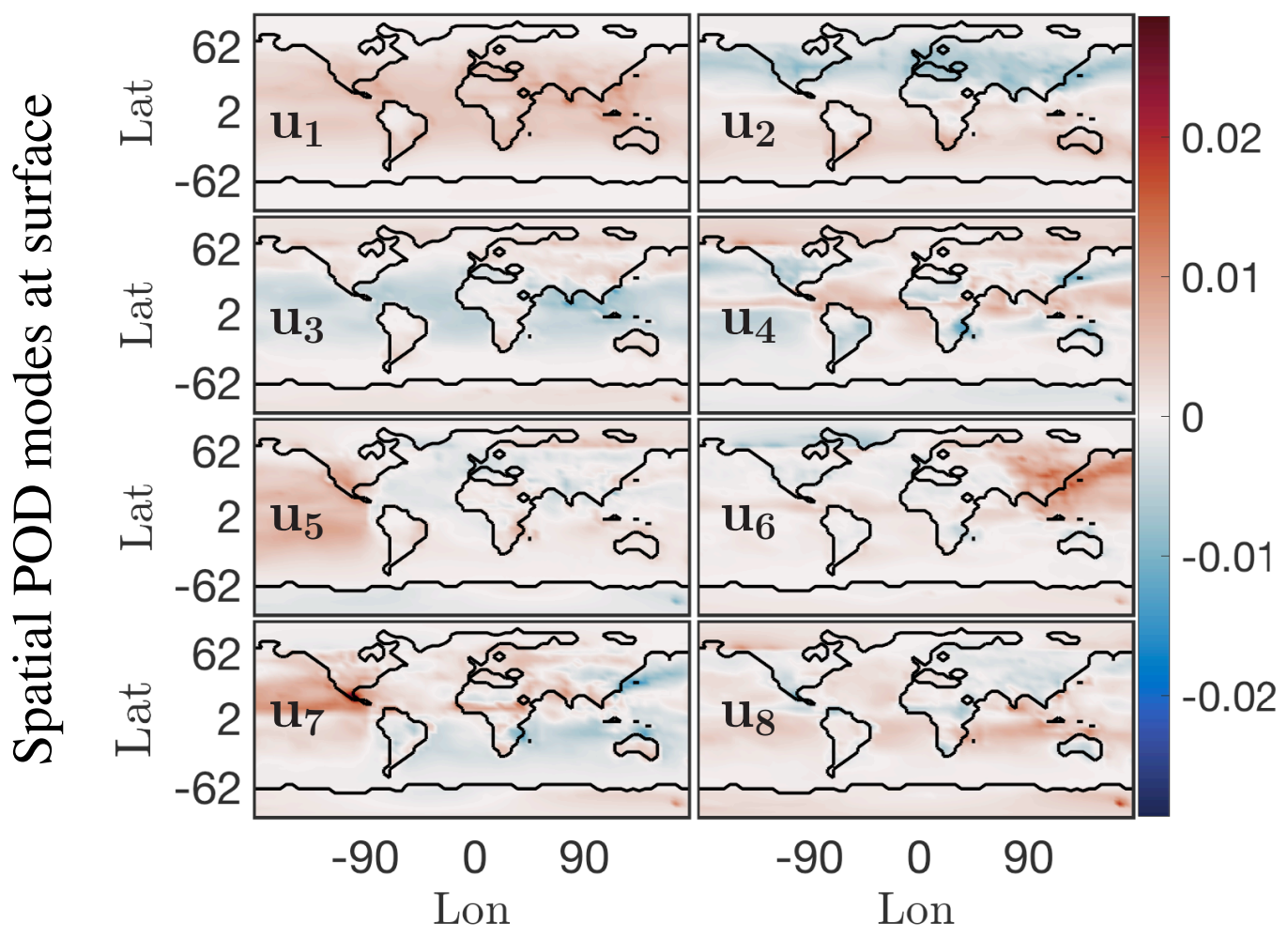

Figure 14. First 8 dominant spatial modes at surface for $\mathbf{O H}$ absolute concentration preprocessed data 

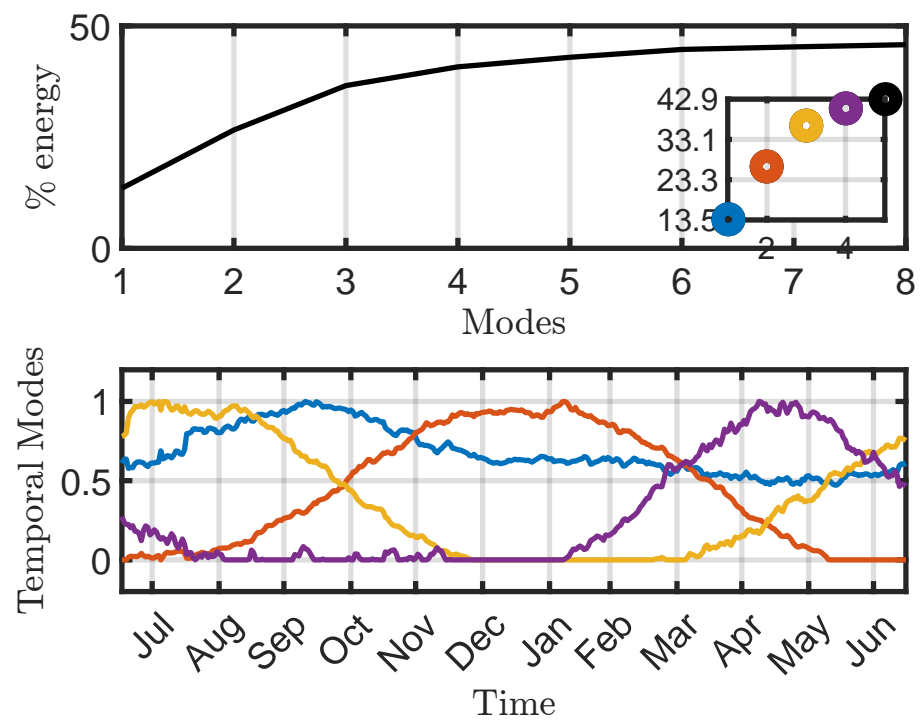

Figure 15. Cumulative energy spectrum from the Nonnegative Matrix Factorization (top) and the corresponding first 4 columns of the ordered $\mathbf{H}$ temporal factor for $\mathbf{O H}$ absolute concentration preprocessed data (bottom).

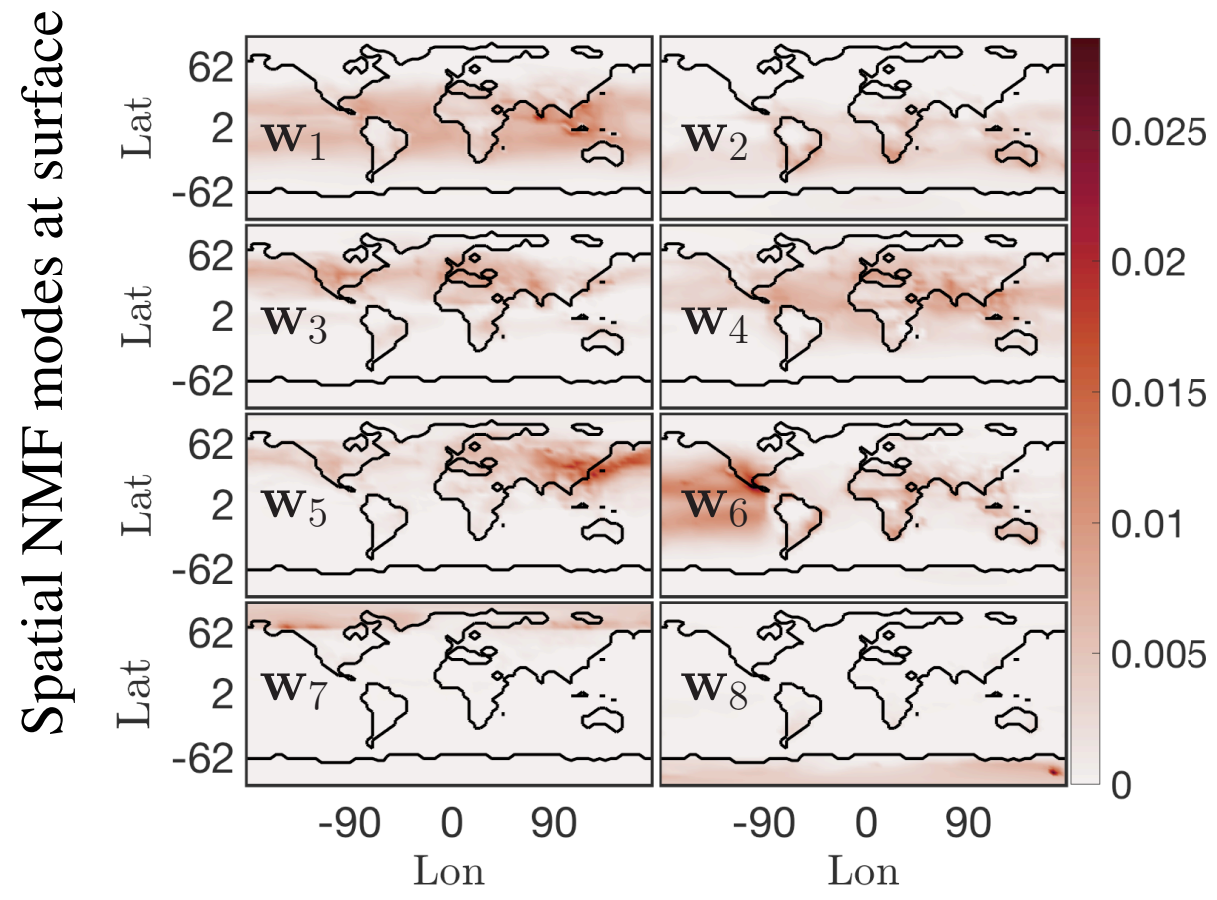

Figure 16. First 8 columns of ordered $\mathbf{W}$ spatial factor from NMF at surface for $\mathbf{O H}$ absolute concentration preprocessed data 

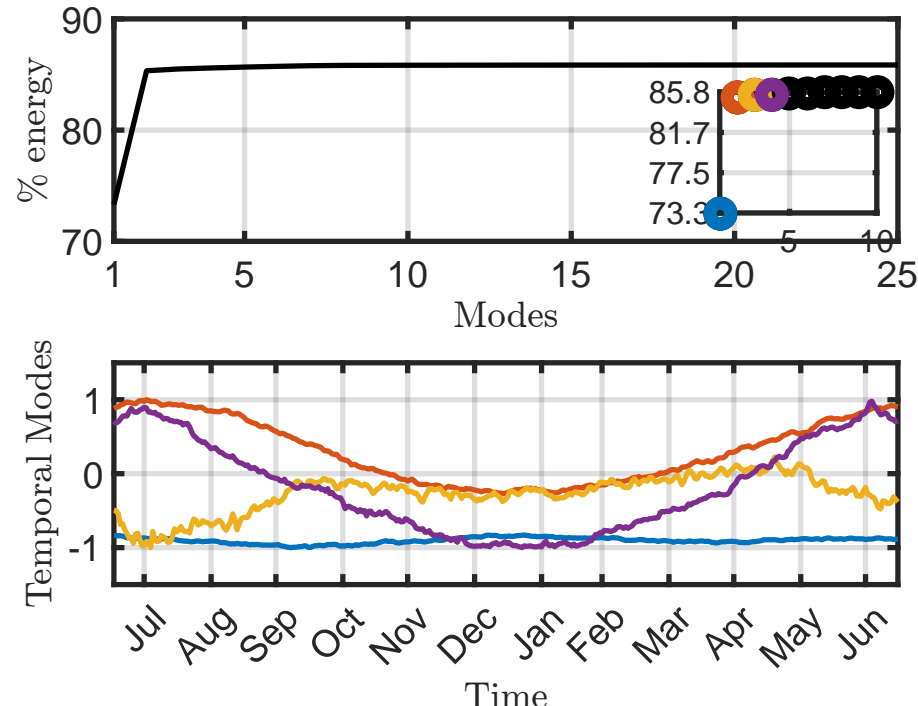

Figure 17. Cumulative energy spectrum from the Sparse Principal Component Anaylsis (top) and the corresponding 4 dominant temporal modes (bottom) for $\mathbf{O H}$ absolute concentration preprocessed data.

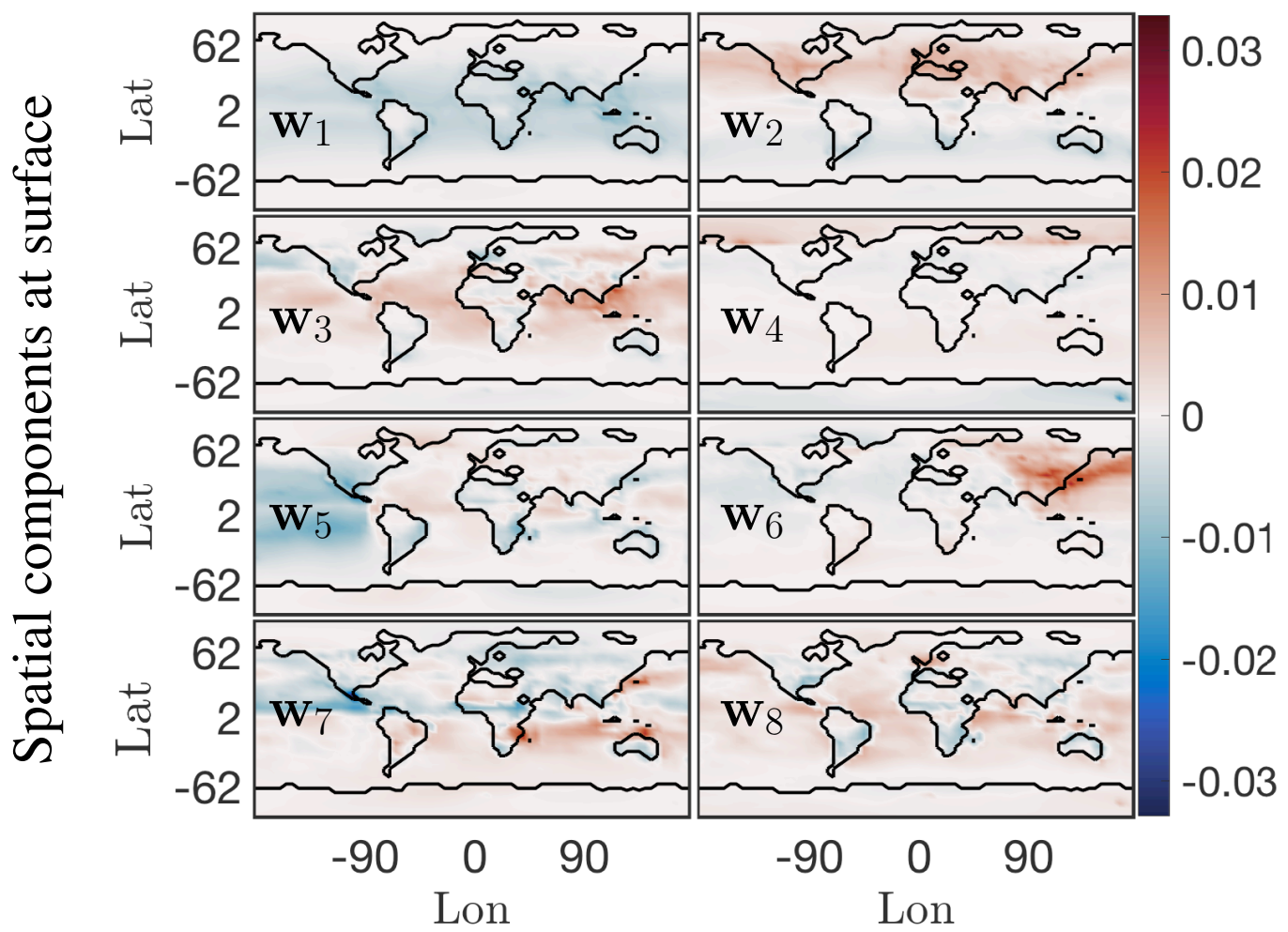

Figure 18. First 8 principal components from SPCA at surface for $\mathbf{O H}$ absolute concentration preprocessed data 

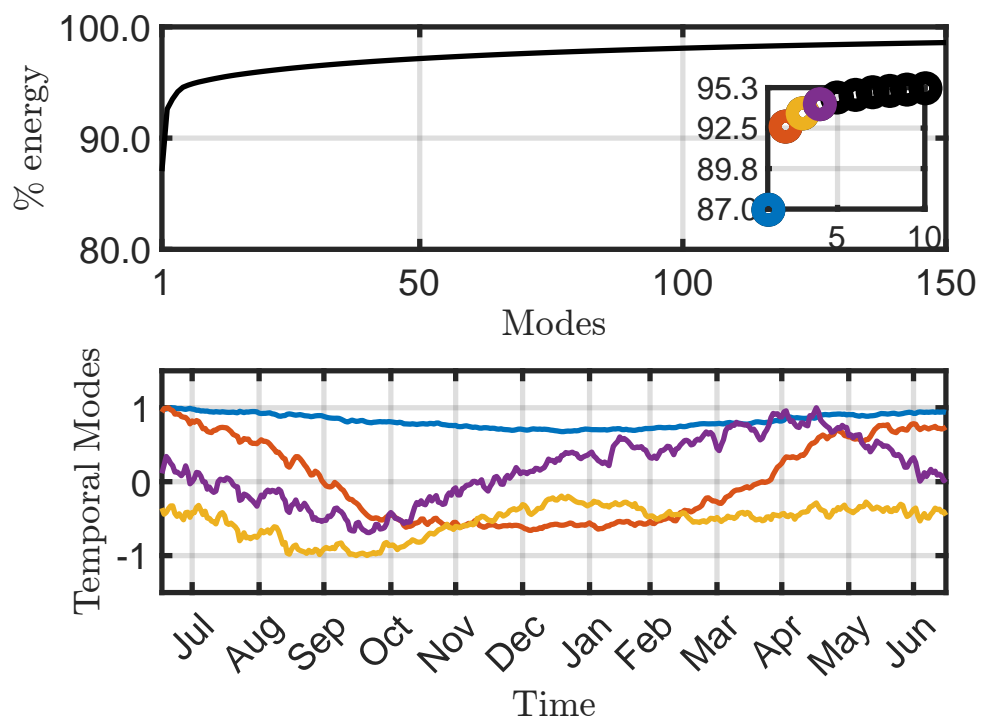

Figure 19. Singular values on a log scale (top) and the corresponding 4 dominant temporal modes (bottom) for log(ISOP) absolute concentration preprocessed data.

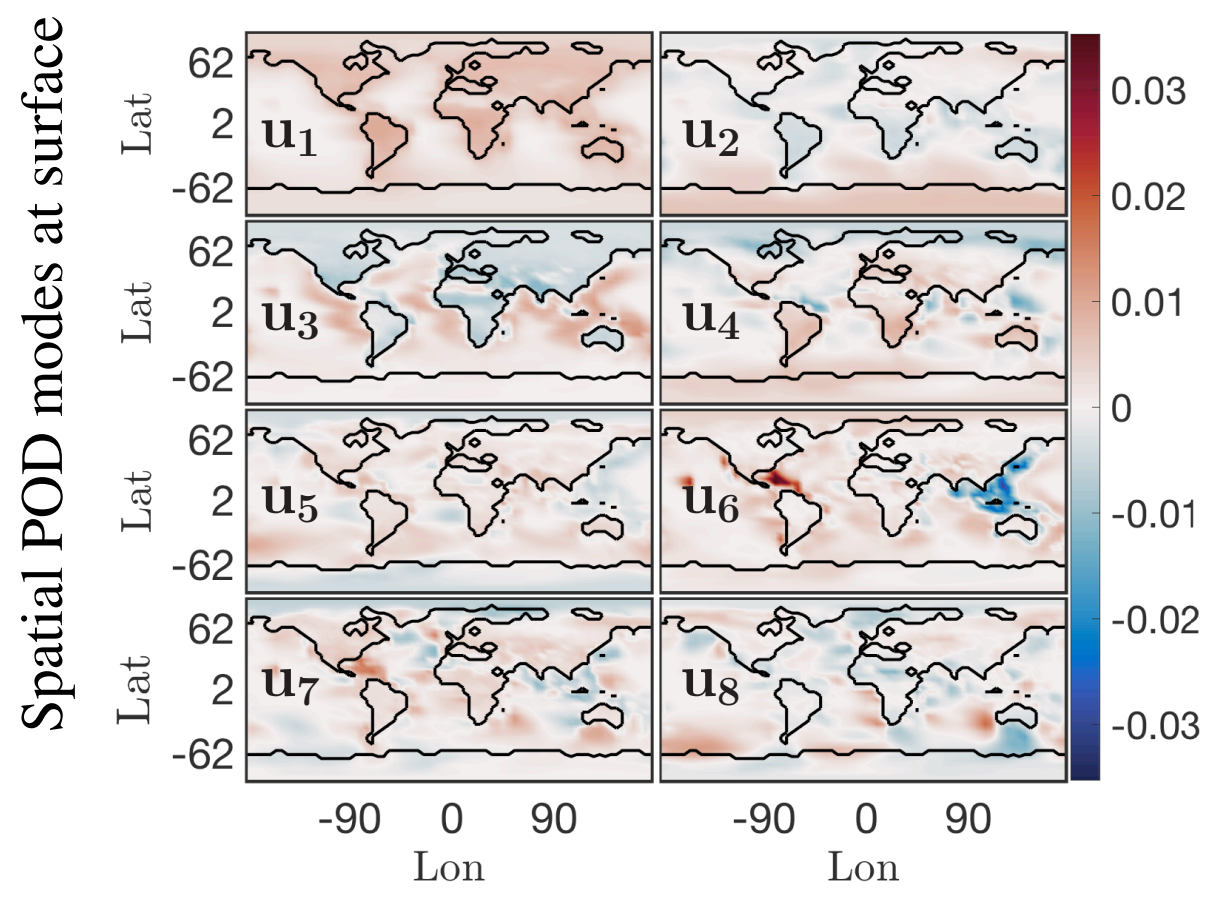

Figure 20. First 8 dominant spatial modes at surface for $\log (\mathbf{I S O P})$ absolute concentration preprocessed data 

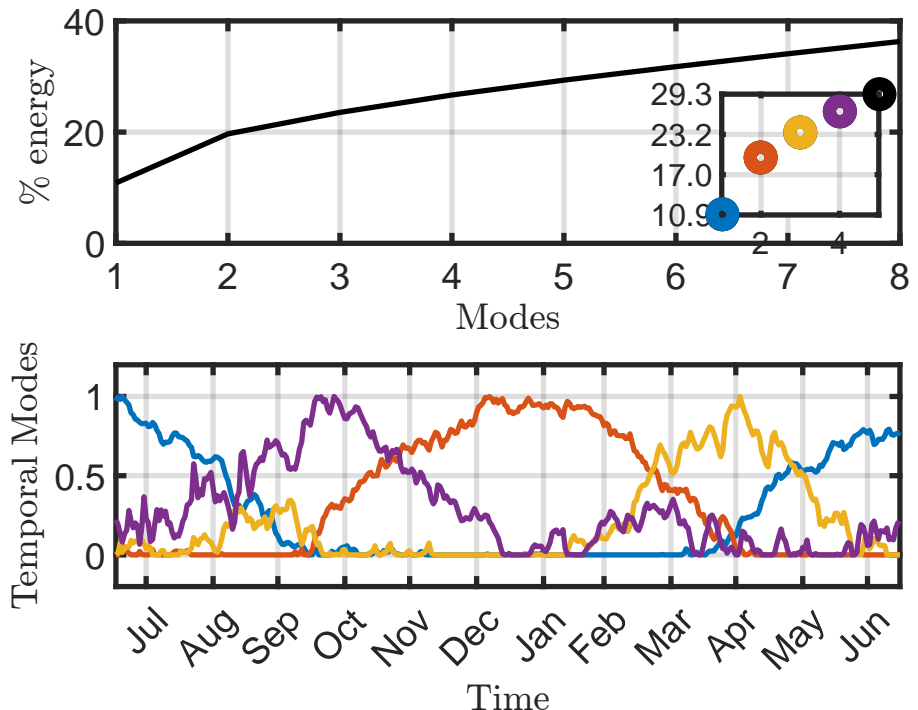

Figure 21. Cumulative energy spectrum from the Nonnegative Matrix Factorization (top) and the corresponding first 4 columns of the ordered $\mathbf{H}$ temporal factor for $\log (\mathbf{I S O P})$ absolute concentration preprocessed data (bottom).

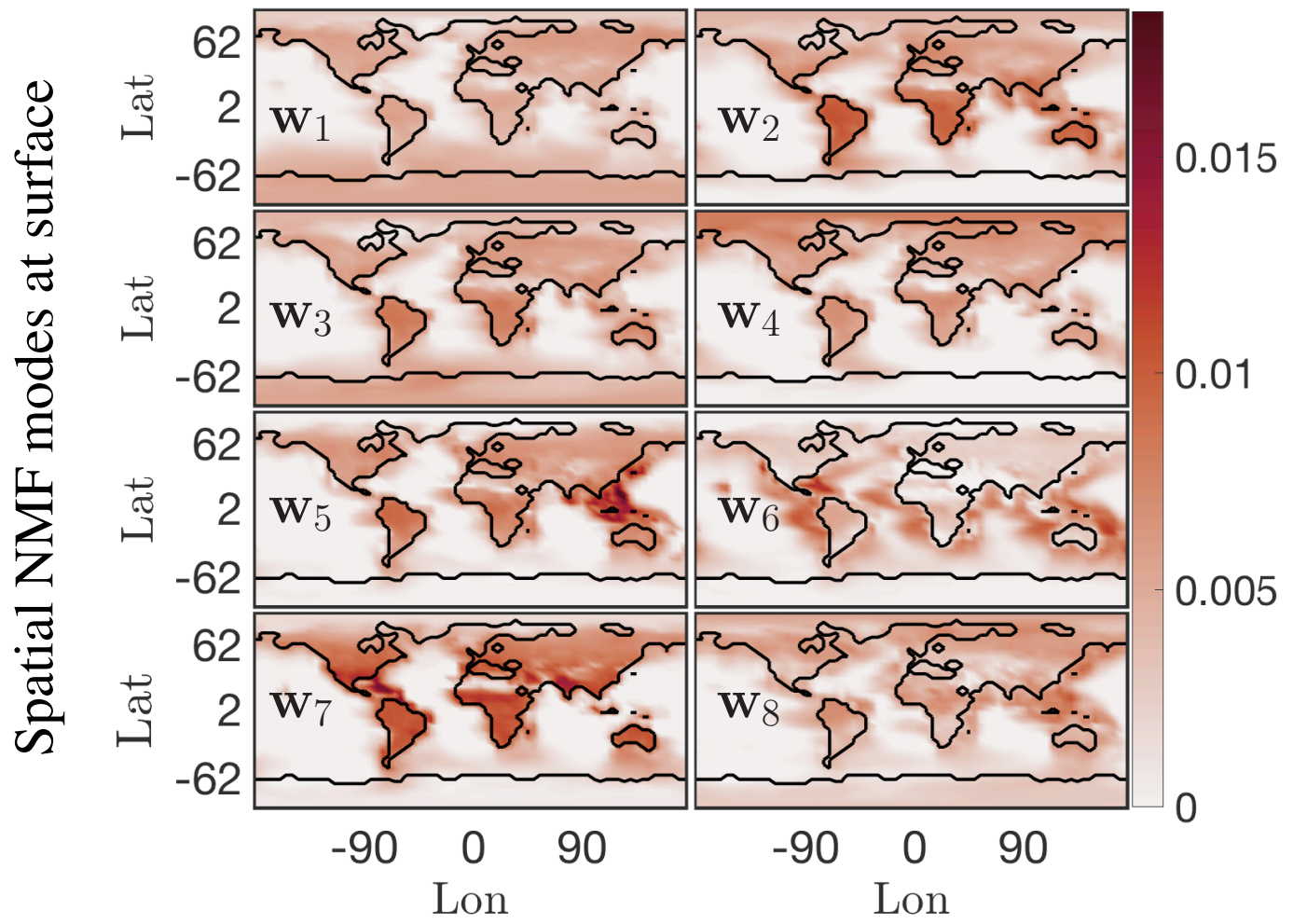

Figure 22. First 8 columns of ordered $\mathbf{W}$ spatial factor from NMF at surface for $\mathbf{l} \mathbf{l}(\mathbf{I S O P})$ absolute concentration preprocessed data 

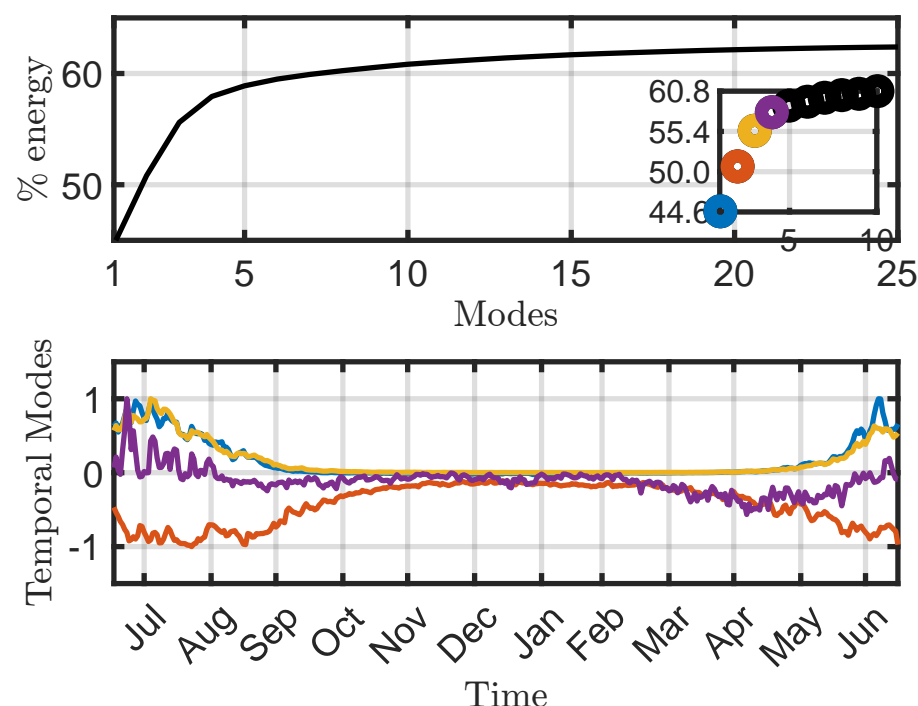

Figure 23. Cumulative energy spectrum from the Sparse Principal Component Anaylsis (top) and the corresponding 4 dominant temporal modes (bottom) for $\log (\mathbf{I S O P})$ absolute concentration preprocessed data.

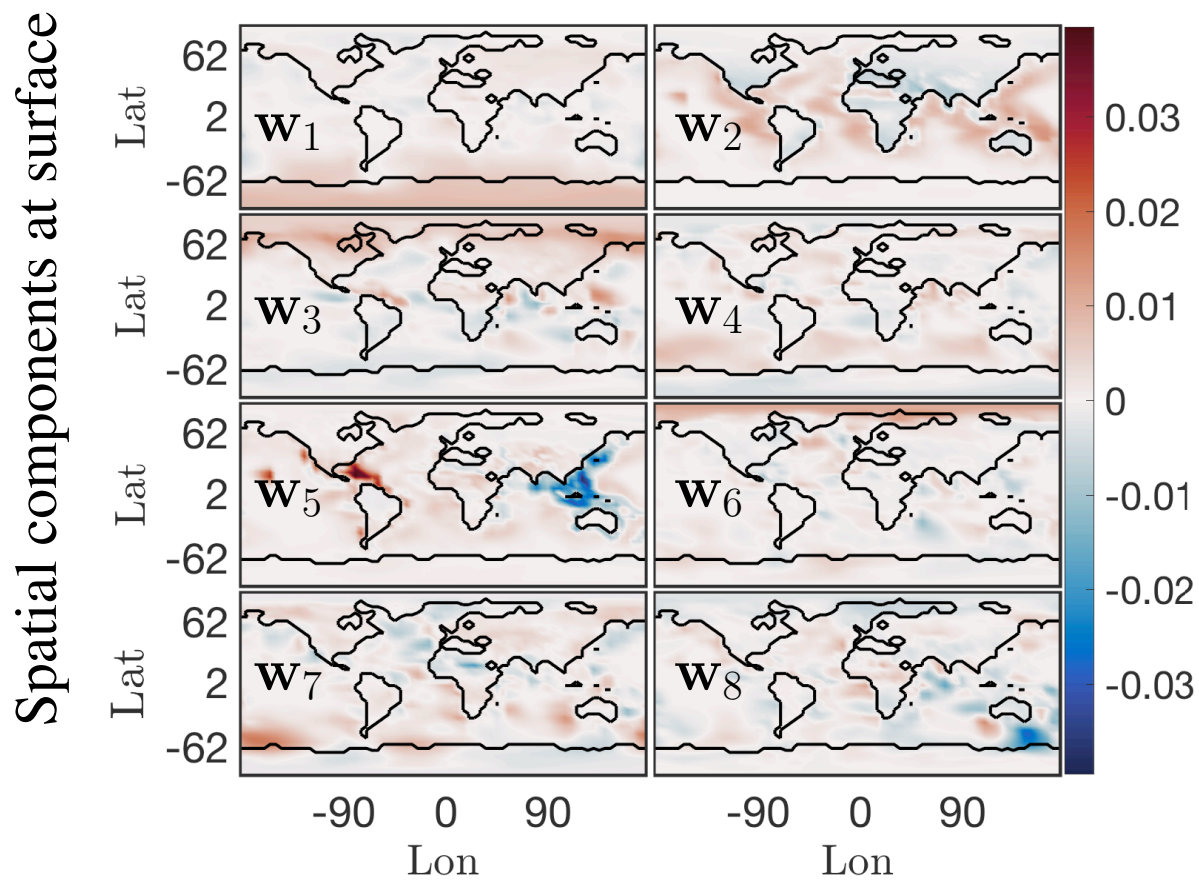

Figure 24. First 8 principal components from SPCA at surface for $\log (\mathbf{I S O P})$ absolute concentration preprocessed data 

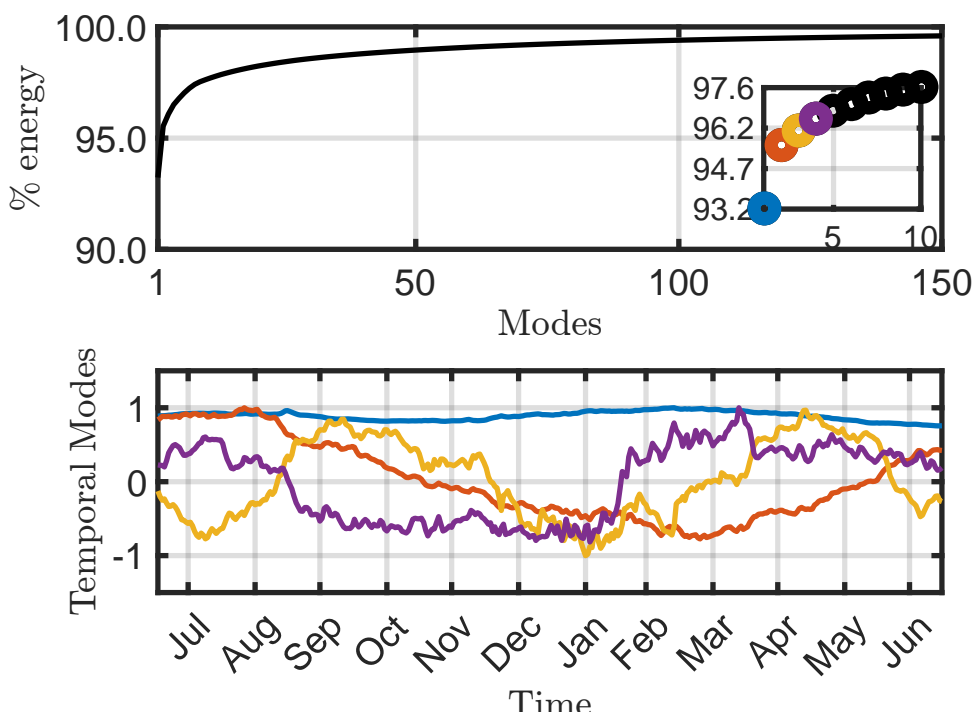

Figure 25. Singular values on a log scale (top) and the corresponding 4 dominant temporal modes (bottom) for CO absolute concentration preprocessed data.

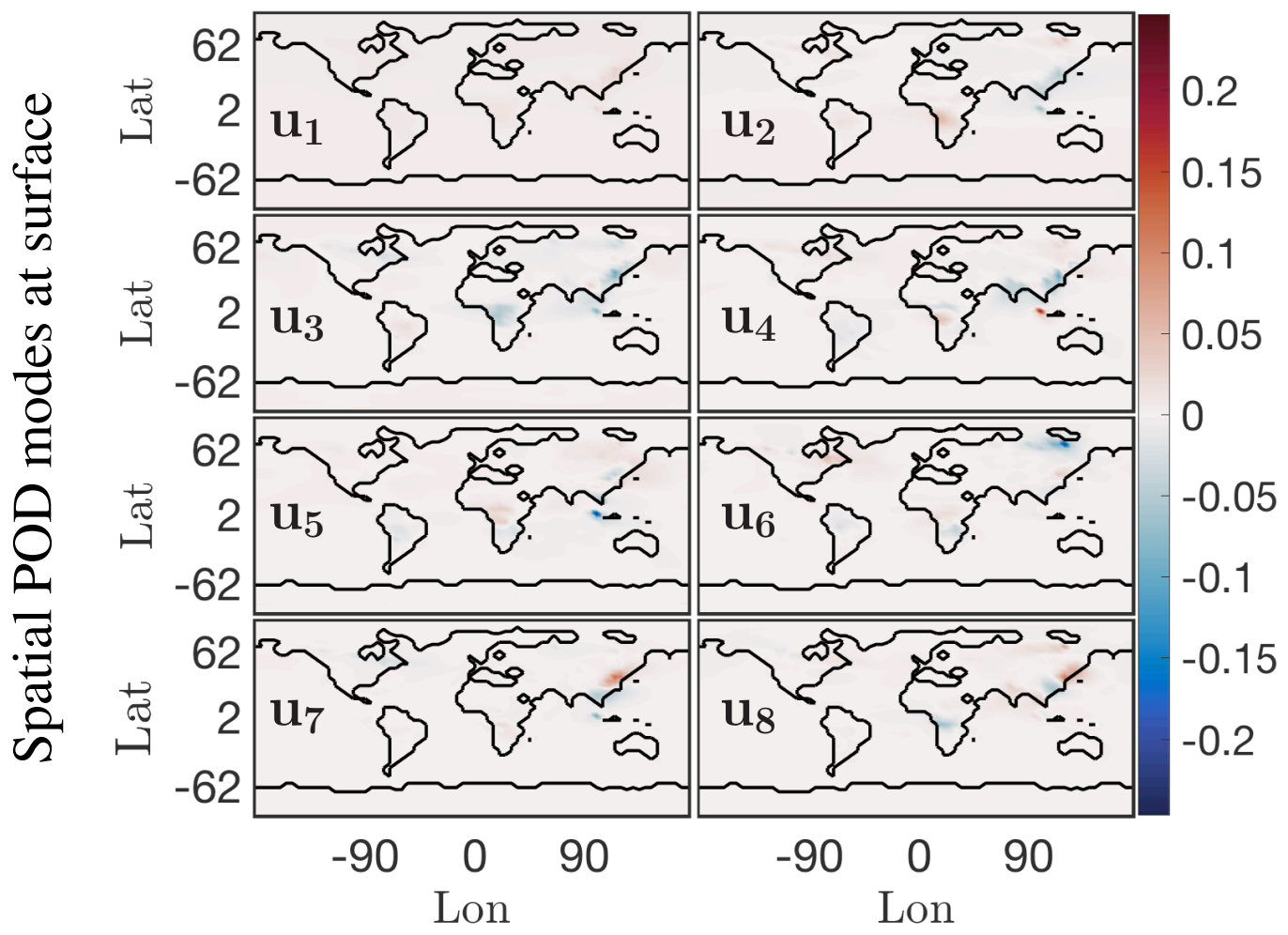

Figure 26. First 8 dominant spatial modes at surface for $\mathbf{C O}$ absolute concentration preprocessed data 

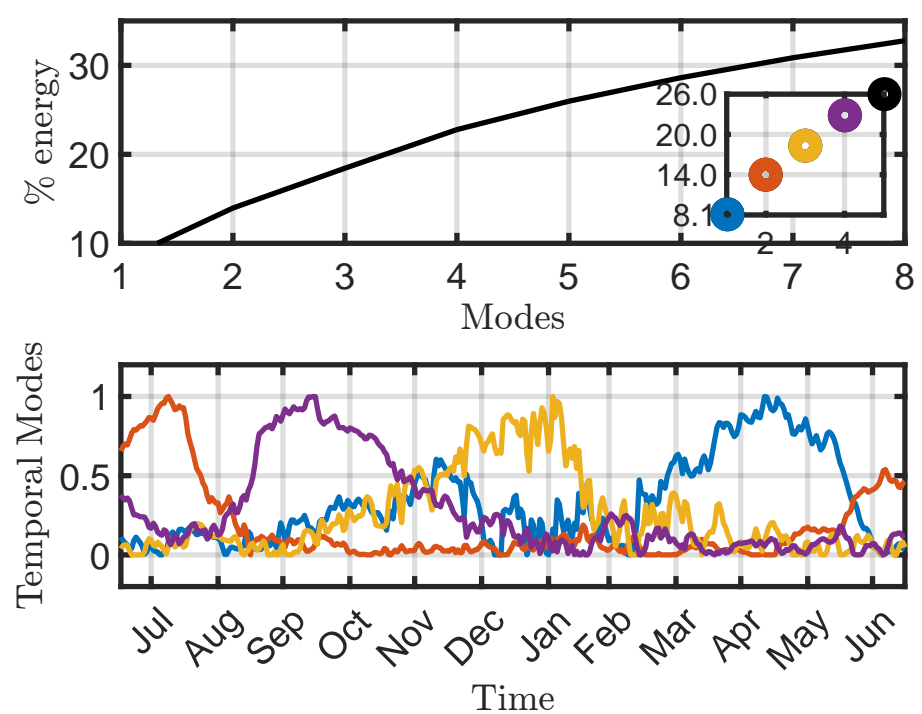

Figure 27. Cumulative energy spectrum from the Nonnegative Matrix Factorization (top) and the corresponding first 4 columns of the ordered $\mathbf{H}$ temporal factor for $\mathbf{C O}$ absolute concentration preprocessed data (bottom).

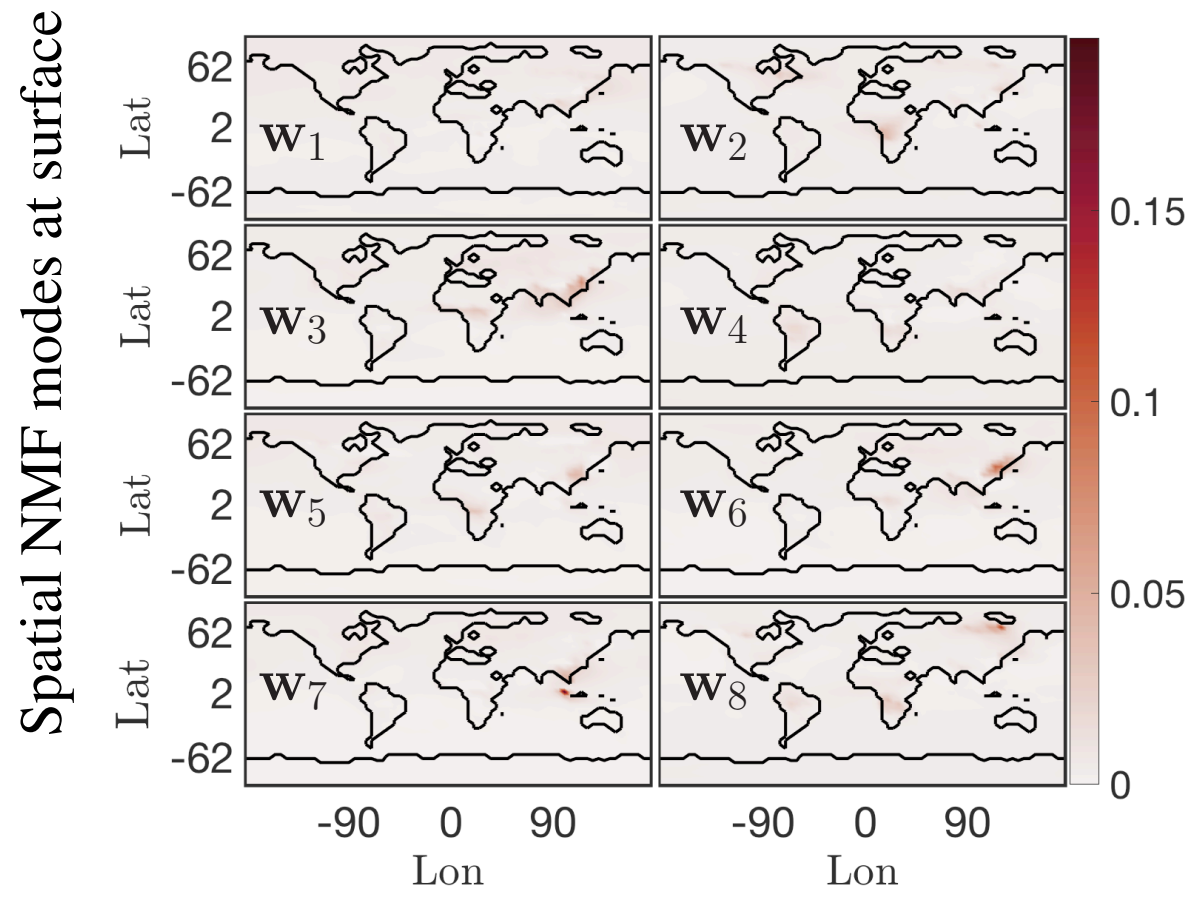

Figure 28. First 8 columns of ordered $\mathbf{W}$ spatial factor from NMF at surface for $\mathbf{C O}$ absolute concentration preprocessed data 

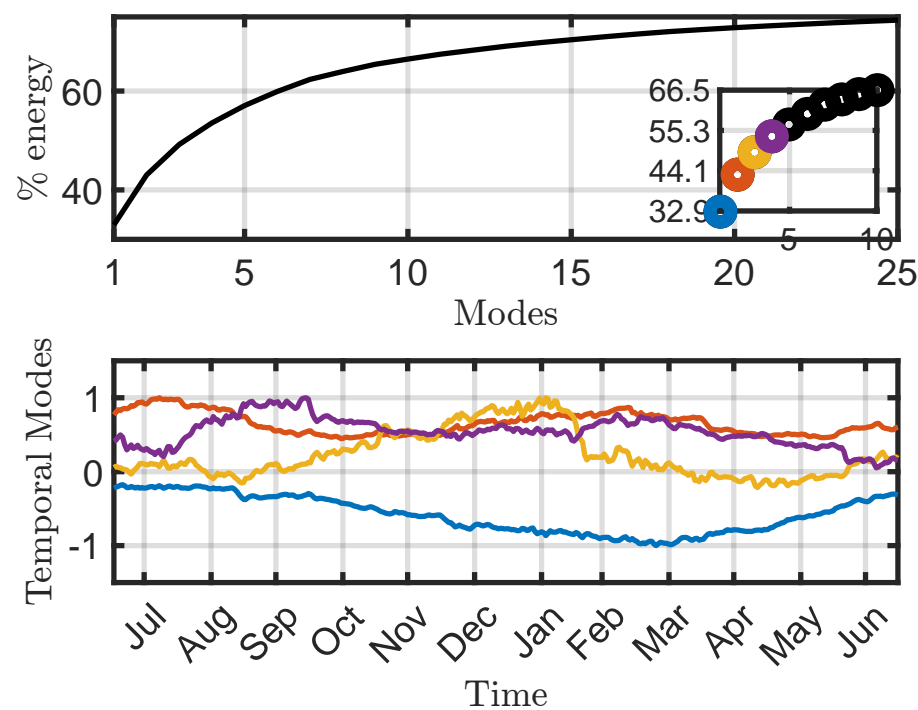

Figure 29. Cumulative energy spectrum from the Sparse Principal Component Anaylsis (top) and the corresponding 4 dominant temporal modes (bottom) for $\mathbf{C O}$ absolute concentration preprocessed data.

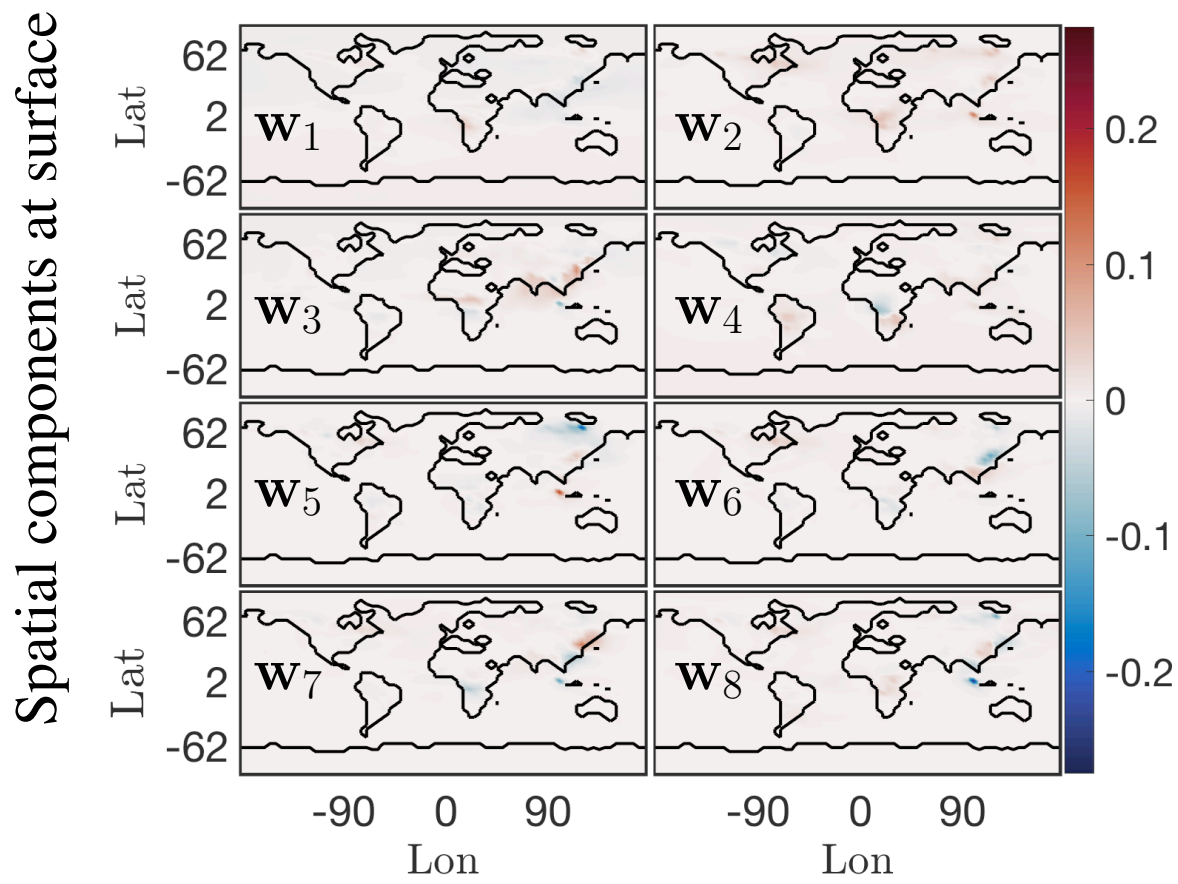

Figure 30. First 8 principal components from SPCA at surface for $\mathbf{C O}$ absolute concentration preprocessed data 